\title{
APPENDIX: METHODIST MISSIONARIES IN UPPER BURMA, 1887-1966
}

\begin{tabular}{|c|c|c|c|}
\hline Surname & $\begin{array}{l}\text { Christian } \\
\text { name }\end{array}$ & Date in Burma & Notes \\
\hline Bateson & Joseph H. & $1886-88$ & Chaplain \\
\hline Winston & W. Ripley & $1887-98$ & Chairman \\
\hline Bestall & Arthur H. & $\begin{array}{l}1887-1910 \\
1920-24\end{array}$ & Chairman \\
\hline Thomas & Thomas W. & $1889-1910$ & \\
\hline Woodward & A. & $1892-1903$ & \\
\hline Sherratt & William & $1896-1933$ & $\begin{array}{l}\text { Bible Society } \\
\text { Secretary }\end{array}$ \\
\hline Phillips & T.G. & 1898-1917 & Chairman \\
\hline Vickers & Agnes $\mathrm{T}$. & 1899-1905 & $\begin{array}{l}\text { WW, married } \\
\text { William Vickery }\end{array}$ \\
\hline Bradford & Edgar J. & 1899-1914 & Acting Chairman \\
\hline Hoyle & Joshua & 1900-2 & \\
\hline Vickery & William & $1903-20$ & \\
\hline Sheldon & Arthur W. & $1903-27$ & Chairman \\
\hline Butt & Miss Nora & 1904-25 & WW \\
\hline Winston & F. Dyson & 1904-13 & \\
\hline Bisseker & Tilden B. & 1905-12 & \\
\hline Hanna & Miss K. & 1907-10 & $\begin{array}{l}\text { WW, married } \\
\text { F.D. Winston }\end{array}$ \\
\hline Chapman & Clement $\mathrm{H}$. & $\begin{array}{l}1908-23 \\
1934-44\end{array}$ & Chairman \\
\hline Walters & H. Crawford & $\begin{array}{l}1910-14 \\
1918-30\end{array}$ & Chairman \\
\hline Moore & Miss H. & $1911-14$ & WW \\
\hline Moore & R. Douglas & $1911-12$ & \\
\hline Winston & Miss J. & 1912-19 & $\begin{array}{l}\text { WW, married } \\
\text { Francis Skinner }\end{array}$ \\
\hline Russell & Maurice H. & $1912-26$ & \\
\hline Underwood & J. England & $1913-22$ & \\
\hline Young & J. Mervyn & $\begin{array}{l}1913-15 \\
1919-33\end{array}$ & Chairman \\
\hline Early & C. Gordon & 1914-15 & \\
\hline Merrick & Miss Anna & $1915-42$ & WW \\
\hline
\end{tabular}




\begin{tabular}{|c|c|c|c|}
\hline Surname & $\begin{array}{l}\text { Christian } \\
\text { name }\end{array}$ & Date in Burma & Notes \\
\hline Adcock & Bert & 1915-19 & \\
\hline Skinner & Francis E. & $1916-29$ & \\
\hline Robertson & Miss Margaret & $1918-46$ & WW \\
\hline Boyce & Mrs. F. & $1920-21$ & WW \\
\hline Hollinshed & Miss Muriel G. & $\begin{array}{l}1920-42 \\
1947-50\end{array}$ & WW \\
\hline Lawley & Miss Lily & $1923-28$ & $\begin{array}{l}\text { WW, married } \\
\text { E.W. Lewis }\end{array}$ \\
\hline Shepherd & H. Vincent & $1924-45$ & Acting Chairman \\
\hline Webb & Paul W. & $1924-36$ & \\
\hline Guyler & $\begin{array}{l}\text { Miss } \\
\text { Fredericka }\end{array}$ & 1925-38 & WW \\
\hline Froud & J. Frank & $1925-28$ & \\
\hline Irvine & J.A.L. & $1926-28$ & \\
\hline Varney & Reginald G. & $1926-55$ & \\
\hline Dawson & Miss Jennie & $1927-31$ & $\begin{array}{l}\text { WW, married } \\
\text { John Leigh }\end{array}$ \\
\hline Willans & H. Crowther & $1927-56$ & $\begin{array}{l}\text { Bible Society } \\
\text { Secretary }\end{array}$ \\
\hline Leigh & John L. & $1928-44$ & \\
\hline Lewis & Ewart W. & 1929-35 & \\
\hline Taylor & George E. & 1929-34 & Lay missionary \\
\hline Firth & G. Eric & $1930-50$ & \\
\hline Reed & Denis & 1930-66 & Chairman \\
\hline Murdoch & Miss Dorothy & 1930 & $\begin{array}{l}\text { WW, married } \\
\text { Denis Reed }\end{array}$ \\
\hline Vincent & Stanley V. & $\begin{array}{l}1930-46 \\
1956-57\end{array}$ & $\begin{array}{l}\text { Bible Society } \\
\text { Secretary }\end{array}$ \\
\hline Smith & Miss Maeve & 1930-33 & WW \\
\hline Hawtin & John G. & 1930-42 & Lay missionary \\
\hline Sewell & Bernard & 1930-45 & \\
\hline Bush & Sister E. & $1932-48$ & $\begin{array}{l}\text { WW, married } \\
\text { Harold Willans }\end{array}$ \\
\hline Topping & Sister L. & $1932-33$ & WW \\
\hline Kinchin & Frederick A. & $\begin{array}{l}1933-41 \\
1946-56\end{array}$ & \\
\hline Holden & William A. & $1934-46$ & \\
\hline Holmes & Arthur J. & $1934-40$ & \\
\hline Gibbons & Sister E. & 1935-39 & \\
\hline
\end{tabular}


APPENDIX

\begin{tabular}{llll} 
Surname & Christian & Date in Burma & Notes \\
& name & & \\
\hline Cleaver & Miss Florence & $1937-66$ & WW \\
& L. & & \\
Acheson & Robert E. & $1937-44$ & \\
Silcock & Valentine & $1937-50$ & \\
Mackley & Miss Dorothy & $1939-42$, & WW \\
& & $1947-59$ & \\
Morgan & Miss Nesta & $1945-60$ & WW \\
Nicholson & Edgar H. & $1947-59$ & \\
Pepper & Anthony T. & $1948-54$ & \\
Musgrave & Miss Hope & $1949-65$ & WW \\
Jones & D. Clifford & $1951-62$ & \\
Broxholme & J. Derek & $1951-62$ & \\
Cowles & Miss E. & $1951-61$ & WW \\
Cowell & Leslie G. & $1951-66$ & \\
Bastin & Miss Margaret & $1953-55$ & WW, married \\
& & & Rev. L.G. Cowell \\
Turtle & T. David & $1953-64$ & \\
Homer & Ivan R. & $1954-65$ & \\
Bain & Miss Ruth & $1955-59$ & WW, married \\
Nurse & Claud A. & $1956-65$ & Ivan Homer \\
Bishop & Edward A. & $1958-65$ & \\
Mann & Ivor F. & $1960-62$ & \\
Pengelly & Miss Mona & $1961-64$ & WW \\
Blakeway- & David G. & $1961-64$ & \\
Smith & & &
\end{tabular}

